\title{
A NOTE ON MACKEY'S IMPRIMITIVITY THEOREM
}

BY PETER R. MUELLER-ROEMER

Communicated by Fred Brauer, May 24, 1971

The imprimitivity theorem-Theorem 2 in [M1]-has many applications, in particular, to quantum mechanics (cf. [M2]). It can be paraphrased as follows:

Every imprimitive (cf. [M1]) unitary representation of a separable locally compact group $G$ is unitarily equivalent to an induced representation.

The importance of this theorem is further reflected in the fact that Fell devotes a sizable part of his recent book [F1] to a generalization of this theorem.

Blattner succeeded in [B1] and [B2] to remove the separability assumption on $G$ and at the same time to simplify the proof of this theorem. In this note we observe how some of the intricacy of Blattner's proof can be reduced to an application of Fubini's theorem.

We are using the same notation as in [B2]. Thus, $H$ is a closed subgroup of the locally compact group $G, \pi$ is the quotient map from $G$ onto the quotient space $G / H$ on which $G$ acts from the right, and $d \xi$ and $d x$ denote (right) Haar measure on $H$ and on $G$ with modular functions $\delta_{H}$ and $\delta_{G}$. The measure $\mu$ on $G \times G$ implicitly defined in Lemma 2 of [B2] can be explicitly defined for $k$ in $C_{0}(G \times G)$ by

$$
\begin{aligned}
& \iint k(y, z) d \mu(y, z) \\
&=\iiint k\left(z y^{-1} \xi^{-1}, y^{-1} \xi^{-1}\right) \delta_{G}^{-1}(\xi y) d \xi d \Lambda(\pi(y), z) .
\end{aligned}
$$

The key formula, in Blattner's proof of Theorem 2,

$$
\int h(x)(f(x), g(x))_{\mu} d x=(P(\tau h) f, f)_{\Lambda},
$$

can now be directly derived as follows: using the definitions of $(\cdot, \cdot)_{\mu}$, $\hat{f}$ and (1) the left-hand side of (2) becomes

AMS 1970 subject classifications. Primary 43A65, 43A20; Secondary 81A54.

Key words and phrases. Imprimitivity, unitary representations, induced representations, locally compact groups, generalized $L^{1}$-algebras.

Copyright (c) American Mathematical Society 1971 


$$
\begin{aligned}
& \iiint \int h(x) \cdot f\left(\pi(x), z y^{-1} \xi^{-1} x\right) \\
& \cdot\left[f\left(\pi(x), y^{-1} \xi^{-1} x\right)\right]^{-1}(\xi y) d \xi d \Lambda(\pi(y), z) d x .
\end{aligned}
$$

By changing the order of integration- $d x$ first-, substituting $y x$ for $x$ and interchanging $d x$ and $d \xi$, the last expression changes to

$$
\iiint h(\pi(y) x) \cdot f(\pi(y) x, z x) \cdot[f(\pi(y) x, x)]^{-} d x d \Lambda(\pi(y), z)
$$

which is equivalent to the right-hand side of (2).

The above observation enabled the author to give a relatively simple proof of the imprimitivity theorem for generalized $L^{1}$-algebras $L(G, A ; E)$ with trivial factor system $E$ but nontrivial action of $G$ on $A$ (cf. [L1] and [L2]) which reads:

To every imprimitive unitary representation $U$ of $L(G, A ; E)$ whose system of imprimitivity is based on $G / H$ there is a unitary representation of $L(H, A ; E)$ such that its induced representation is unitarily equivalent to $U$.

The author would like to express his gratitude to Horst Leptin for posing this problem to him.

\section{REFERENCES}

[B1] R. J. Blattner, On induced representations, Amer. J. Math. 83 (1961), 79-98. MR 23 \#A2757.

[B2] - Positive definite measures, Proc. Amer. Math. Soc. 14 (1963), 423-428. MR 26 \#5095.

[F1] J. M. G. Fell, An extension of Mackey's method to Banach *algebraic bundles, Mem. Amer. Math. Soc. No. 90 (1969). MR 41 \#255.

[L1] H. Leptin, Verallgemeinerte $L^{1}$-Algebren und projektive Darstellungen lokal kompakter Gruppen. I, II, Invent. Math. 3 (1967), 257-281; ibid. 4 (1967), 68-86. MR 37 \#5328.

[L2] - Darstellungen verallgemeinerter L'-Algebren. I, Invent. Math. 5 (1968), 192-215. MR 38 \#5022.

[M1] G. W. Mackey, Imprimitivity for representations of locally compact groups. I, Proc. Nat. Acad. Sci. U.S.A. 35 (1949), 537-545. MR 11, 158.

[M2] ——, Induced representations of groups and quantum mechanics, Benjamin, New York; Editore Boringhieri, Torino, 1968.

University of Heidelberg, Heidelberg, Germany

East Carolina University, Greenville, North Carolina 27834 